\title{
Synthesis and Condensation of 3-(Triethoxysilyl)propyl-Terminated Polystyrene
}

\author{
Keita SuzUKI, Jun-ichi OKU, ${ }^{\dagger}$ Mikio TAKAKI, Hirofumi OKaBAYASHI, \\ and Charmian J. O'CONNOR* \\ Department of Applied Chemistry, Nagoya Institute of Technology, \\ Gokiso-cho, Showa-ku, Nagoya 466-8555, Japan \\ *Department of Chemistry, The University of Auckland, \\ Private Bag 92019, Auckland, New Zealand
}

(Received June 17, 2003; Accepted September 27, 2003)

\begin{abstract}
A well-defined polymeric silane coupling agent, 3-(triethoxysilyl)propyl-terminated polystyrene (TESiP-PS) was synthesized by reaction of polystyryllithium with (3-chloropropyl)triethoxysilane. The reaction is greatly affected by reaction conditions. The yield of TESiP-PS was quantitative in a benzene/tetrahydrofuran medium at $0{ }^{\circ} \mathrm{C}$, whereas it was as low as $5 \%$ in benzene at room temperature. TESiP-PS obtained under the former conditions has $95 \%$ functionality and a monodispersity of 1.05 . Condensation of TESiP-PS proceeds quantitatively in the presence of either base or acid catalyst. This result is different from that for triethoxysilyl-terminated polystyrene (TESi-PS), which undergoes complete cleavage in the presence of base catalysts. This sharp difference indicates that the introduction of a trimethylene group between the trimethoxysilyl group and polystyrene effectively suppressed the cleavage reaction.

KEY WORDS 3-(Triethoxysilyl)propyl-Terminated Polystyrene / Living Polystyrene / Condensation / Spacer / Base Catalyst / Cleavage Reaction /
\end{abstract}

It is an interesting subject for material technology to improve the performance of a polymer material by hybridizing it with an inorganic substance. The inorganic substance is usually employed as a filler and is often treated with a surface treatment agent to enhance its additional effect on intended properties such as compatibility, strength, or loss factor related to damping property. A variety of silane coupling agents, most of which are relatively low molecular weight compounds, ${ }^{1}$ has thus far been developed for these purposes.

Recently, the reaction of alkoxysilyl-functionalized polymers with tetra-alkoxysilyl monomers has been used as the basis for production of hybrid materials. Long et ll. $^{2}$ reported the successful preparation of the oligomers of phenyltrimethoxysilyl-terminated polystyrene by conventional anionic techniques. They showed that mono-functionalized oligomers are useful as stable precursors for the preparation of soluble starshaped polymers.

We have studied the synthesis and condensation of the well-defined triethoxysilyl-terminated polystyrene (TESi-PS) $)^{3-6}$ as a control for polymeric silane coupling agents. TESi-PS thus prepared was quantitatively condensed by various catalysts. ${ }^{6}$ However, in the presence of strong base catalysts, ${ }^{5}$ we found that optimum yield of condensed TESi-PS was followed by its decomposition with the passage of time to a compound having the same molecular weight as that of TESi-PS.
This sequence was due to a cleavage reaction that involved elimination of polystyrene moieties from condensed TESi-PS; namely, scission of a benzyl-Si bond of TESi-PS. It then becomes apparent that this cleavage reaction would be suppressed by introducing an alkyl group as spacer between a triethoxysilyl group and polystyrene moiety.

In this present study, synthesis and condensation of 3-(triethoxysilyl)propyl-terminated polystyrene (TESiP-PS) was carried out with two aims. One was to develop a well-defined polymeric silane coupling agent that can undergo condensation without side reactions even in the presence of base catalysts. The other was to clarify further the mechanism of the cleavage reaction in the case of base catalysts.

\section{EXPERIMENTAL}

\section{Materials}

(3-Chloropropyl)triethoxysilane (CPTESi; ShinEtsu Silicon Chemicals) was dried on a vacuum line over calcium hydride for $24 \mathrm{~h}$, and then distilled. secButyllithium (Aldrich, 1.3 M in cyclohexane) was used after careful filtration and solvent replacement with benzene. Styrene was purified by the method described in the previous paper. ${ }^{5}$ Benzene and tetrahydrofuran (THF) were purified according to the conventional living anionic polymerization technique. TESi-PS

${ }^{\dagger}$ To whom correspondence should be addressed (E-mail: j-oku@nitech.ac.jp). 
was prepared by the coupling reaction of PStLi with CTES, as previously reported. ${ }^{3}$ Catalysts used for condensation, such as methanesulfonic acid, $\mathrm{LiOH}$, $\mathrm{NaOH}$, and $\mathrm{KOH}$, were reagent grade and were used as received.

\section{Methods}

Coupling reactions of PStLi with CPTESi were performed by dropwise addition of a benzene solution of PStLi to a solution of CPTESi in benzene or THF. All operations relating to this reaction were carried out under high vacuum in an all-glass apparatus equipped with breakseals. The resulting polymers were recovered by the method described in the previous paper $^{3}$ for recovering polystyrene.

The condensation of TESiP-PS or TESi-PS, using methanesulfonic acid as catalyst proceeded throughout in a homogeneous system in THF in a sealed ampoule at $60^{\circ} \mathrm{C}$. In the presence of alkali hydroxide catalysts, the condensation was carried out as follows. Since alkali hydroxides are insoluble in THF, an aqueous solution of hydroxide was added to a THF solution of TESiPS or TESiP-PS in an ampoule, and the heterogeneous reaction mixture was stirred at $60^{\circ} \mathrm{C}$ for the prescribed time. The resulting polymer was recovered by pouring the reaction mixture into a large excess of methanol.

The extent of conversion of TESiP-PS or TESi-PS to the oligomer was determined from the gel permeation chromatography (GPC) curve of the polymer recovered after condensation, i.e., from the ratio of the peak area of condensed compounds to the total response, as obtained using an RI detector.

\section{Characterization}

GPC curves were recorded on a Toso HLC-802UR with $\mathrm{G} 2000 \mathrm{H}$ and $\mathrm{G} 3000 \mathrm{H}$ columns or on a Toso HLC$802 \mathrm{~A}$ with two GMH columns at a column oven temperature of $40{ }^{\circ} \mathrm{C}$. THF was used as the eluent and the flow rate was $1 \mathrm{~mL} \mathrm{~min}^{-1}$. The GPC data were calibrated with polystyrene standards (Figure 1 ). ${ }^{1} \mathrm{H}$ NMR spectra were obtained with a Varian XL-200 spectrom- eter. The solvent used was chloroform- $d$ containing $1 \%$ tetramethylsilane as internal reference.

\section{RESULTS AND DISCUSSION}

\section{Synthesis of TESiP-PS}

To prepare well-defined TESiP-PS, the coupling reaction of PStLi with CPTESi was carried out under reaction conditions that were focused on the reaction medium, since it has been reported that this type of nucleophilic substitution is highly affected by reaction medium. ${ }^{7-10}$ The functionalities of the prepolymers (TESiP-PS) thus obtained were determined by carrying out their condensation reactions in the presence of sodium hydroxide as catalyst. The results are shown in Table I. The coupling reaction in a benzene/THF medium at $0{ }^{\circ} \mathrm{C}$ afforded quantitatively TESiP-PS with more than $94 \%$ functionality (f) and a monodispersity of $M_{\mathrm{w}} / M_{\mathrm{n}}=1.05$ (codes 1 and 2, Table I).

In contrast, the coupling reaction in a benzene medium at room temperature gave TESiP-PS in low yield, as shown for codes 3 and 4, Table I, though the reaction of PStLi with CTES afforded TESi-PS ${ }^{3}$ quan-

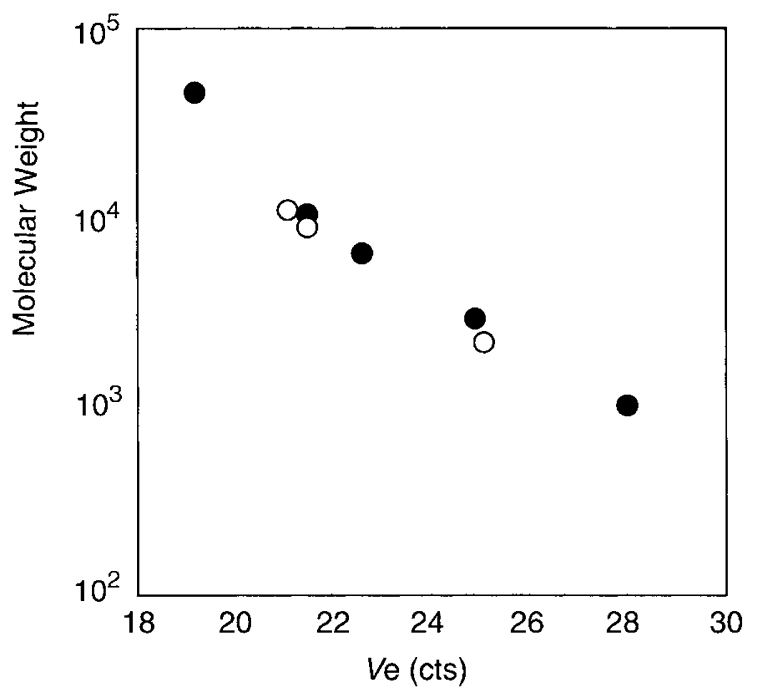

Figure 1. GPC calibration curves (elution volume ( $V \mathrm{e}$, counts (cts)) vs. molecular weight): polystyrene standards $(\bullet)$ and TESiPPS polymer (O).

Table I. Preparation of TESiP-PS by coupling PSt-Li with CPTESi

\begin{tabular}{ccccccccccc}
\hline & \multicolumn{2}{c}{ PSt-Li soln } & \multicolumn{2}{c}{ CPTESi soln } & \multicolumn{2}{c}{ coupling reaction } & \multicolumn{2}{c}{ TESiP-PS } \\
\hline code & solvent & $\begin{array}{c}{[\mathrm{LE}]^{\mathrm{b}}} \\
\left(\mathrm{mmol} \mathrm{L}^{-1}\right)\end{array}$ & solvent & $\begin{array}{c}{[\mathrm{CPTESi}]} \\
\left(\mathrm{mmol} \mathrm{L}^{-1}\right)\end{array}$ & $\begin{array}{c}{[\mathrm{Cl}] /[\mathrm{LE}]} \\
\text { temp } \\
\left({ }^{\circ} \mathrm{C}\right)\end{array}$ & $\begin{array}{c}\text { THF } \\
(\mathrm{vol} \mathrm{\% )}\end{array}$ & $M_{\mathrm{n}} \times 10^{-3}$ & $M_{\mathrm{w}} / M_{\mathrm{n}}$ & $\mathrm{f}^{\mathrm{c}}$ \\
\hline 1 & benzene & 48.6 & THF & 167.1 & 3.44 & 0 & 26.5 & 2.20 & 1.05 & 96 \\
2 & benzene & 48.3 & THF & 169.5 & 3.51 & 0 & 26.9 & 2.20 & 1.05 & 94 \\
3 & benzene & 29.8 & benzene & 94.0 & 3.15 & r.t. & 0 & 2.20 & 1.05 & 5 \\
4 & benzene & 19.0 & benzene & 49.1 & 2.58 & r.t. & 0 & 3.20 & 1.03 & 5 \\
\hline
\end{tabular}

${ }^{\mathrm{a}}$ Reaction time, $2 \mathrm{~h} .{ }^{\mathrm{b}}$ Molar concentration of the living end. ${ }^{\mathrm{c}}$ Functionality ( $\mathrm{f}$ (the maximum value of $\mathrm{Y}$ ), $\mathrm{Y}(\%)=$ $100 \times$ [the GPC band area of polymerized TESiP-PS]/[the total GPC band area of the reacted products]) determined from the condensation of coupling reaction product in the presence of $\mathrm{NaOH}$. 
Table II. Condensation of the prepolymers (codes 3 and 4 in Table I) using acid or base catalyst using various conditions ${ }^{\mathrm{a}}$

\begin{tabular}{|c|c|c|c|c|c|c|}
\hline \multirow[t]{2}{*}{ run } & \multicolumn{2}{|c|}{ prepolymer } & \multicolumn{2}{|c|}{ catalyst } & \multirow{2}{*}{$\begin{array}{l}\text { time } \\
\text { (h) }\end{array}$} & \multirow{2}{*}{$\begin{array}{c}\text { condensate yield } \\
(\%)\end{array}$} \\
\hline & code & $\left(\mathrm{mmol} \mathrm{kg}^{-1}\right)$ & & $\left(\mathrm{mol} \mathrm{kg}^{-1}\right)$ & & \\
\hline 1 & 3 & 19.8 & $\mathrm{CH}_{3} \mathrm{SO}_{3} \mathrm{H}$ & 0.10 & 72 & 82 \\
\hline 2 & 4 & 40.1 & $\mathrm{CH}_{3} \mathrm{SO}_{3} \mathrm{H}$ & 0.10 & 48 & 79 \\
\hline 3 & 3 & 19.8 & $\mathrm{NaOH}$ & 0.95 & 72 & 5 \\
\hline 4 & 4 & 19.0 & $\mathrm{NaOH}$ & 0.99 & 72 & 5 \\
\hline
\end{tabular}

${ }^{\mathrm{a}}$ Temperature, $60^{\circ} \mathrm{C}$; solvent, THF.

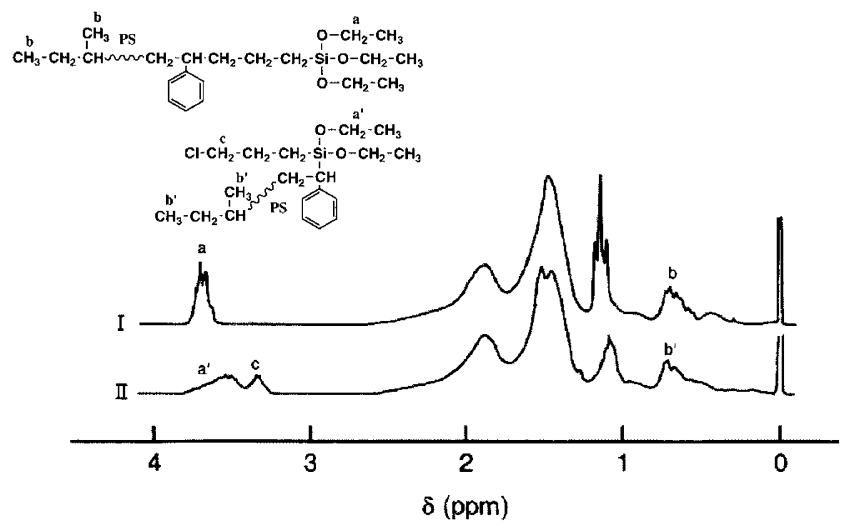

Figure 2. ${ }^{1} \mathrm{H}$ NMR spectra of the coupling products of PSt-Li with CPTESi in benzene/THF and benzene: spectra I and II are of those in codes 1 and 3 in Table I, respectively.

titatively under similar conditions. In order to clarify the reason for the very low yield of TESiP-PS, an alternative method was employed to determine the functionalities of reaction products in the benzene medium; i.e., the condensation of prepolymers (codes 3 and 4) were carried out using methanesulfonic acid as catalyst instead of sodium hydroxide. As shown in Table II, yields of condensates of prepolymers under acidic conditions were about $80 \%$, indicating that at least $80 \%$ of the polystyrene molecules have functional groups susceptible to condensation by the acid catalyst.

The sharp contrast between the extent of condensation by acid catalysts and that by base catalysts may result from the structure of the functional groups. Possible functional groups, which are formed in the coupling reaction of PStLi with CPTESi, are a 3-(triethoxysilyl)propyl group and a (3chloropropyl)diethoxysilyl group. To confirm the functional group, ${ }^{1} \mathrm{H}$ NMR measurements were taken for representative prepolymers (codes 1 and 3, Table I). Figure 2 shows the NMR spectra of the prepolymers. In spectrum I (code 1), the signal of the methylene protons (a) of the ethoxy group is observed from 3.62 to $3.77 \mathrm{ppm}$, and the methyl protons (b) of the initiator fragment (sec-butyl) can be seen from 0.58 to $0.80 \mathrm{ppm}$. The peak area ratio of the methylene protons to the methyl protons (i.e., a : b) was evaluated as 5.91 (theor, 6) : 6. This value shows that the functionality of the 940

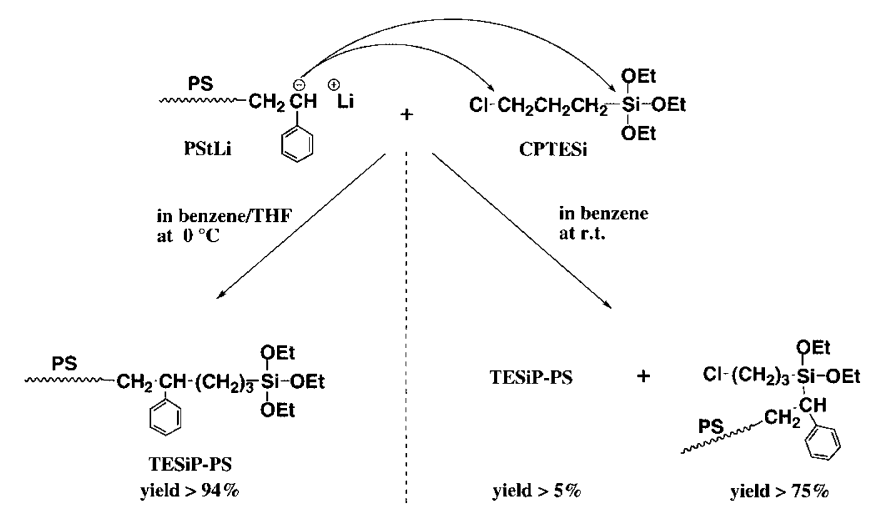

Scheme 1. Reactions of PStLi with CPTESi in bezene/THF and benzene.

code 1 prepolymer is $98 \%$. In spectrum II, the peak c around $3.35 \mathrm{ppm}$ comes from the chloromethylene signal of the code 3 prepolymer. The ratio of the peak areas of the chloromethylene protons to methyl protons b' was estimated to be $1.77: 6$, showing that (3chloropropyl)diethoxysilyl groups were introduced at the end of polystyrene giving $89 \%$ functionality to the code 3 prepolymer. These ${ }^{1} \mathrm{H}$ NMR functionality values are somewhat higher than those obtained after condensation in the presence of acid catalyst. The difference may be due to the incompleteness of the condensation process and / or to the precision of ${ }^{1} \mathrm{H}$ NMR analyses.

In conclusion, results for the synthesis of TESiPPS under two different reaction conditions are summarized in Scheme 1. With benzene/THF as the medium and a temperature of $0{ }^{\circ} \mathrm{C}$, the reaction of PStLi with CPTESi affords quantitatively well-defined TESiP-PS. With benzene as medium and at room temperature, the yield of TESiP-PS is as low as $5 \%$, while (3chloropropyl)diethoxysilyl-terminated polystyrene is obtained as a major product in a yield of $75 \%(89 \%$ by ${ }^{1} \mathrm{H}$ NMR).

\section{Condensation of TESiP-PS.}

TESiP-PS and TESi-PS (for purposes of comparison) were subjected to condensation using base catalysts. Characteristics of TESiP-PS and TESi-PS were as follows. TESiP-PS: $M_{\mathrm{n}}=2.10 \times 10^{3}, M_{\mathrm{w}} / M_{\mathrm{n}}=1.05, f=$ $96 \%$. TESi-PS: $M_{\mathrm{n}}=7.60 \times 10^{3}, M_{\mathrm{w}} / M_{\mathrm{n}}=1.06, f=$ 
Table III. Condensations of TESiP-PS and TESi-PS in the presence of base catalysts ${ }^{\mathrm{a}}$

\begin{tabular}{ccccrrrrr}
\hline run & $\begin{array}{c}\text { prepolymer } \\
\left(\mathrm{mmol} \mathrm{kg}^{-1}\right)\end{array}$ & \multicolumn{2}{c}{ catalyst } & time & \multicolumn{3}{c}{ condensate } \\
& & & $\left(\mathrm{mol} \mathrm{kg}^{-1}\right)$ & $(\mathrm{h})$ & yield $^{\mathrm{b}}(\%)$ & $\mathrm{DP}^{\mathrm{c}}$ & $M_{\mathrm{w}} / M_{\mathrm{n}}$ \\
\hline TESiP-PS & & & & & & & & \\
1 & 20.0 & $\mathrm{LiOH}$ & 0.99 & 1 & 94 & 5 & 1.07 \\
2 & 20.1 & $\mathrm{LiOH}$ & 0.98 & 2 & 93 & 5 & 1.06 \\
3 & 20.0 & $\mathrm{LiOH}$ & 1.00 & 168 & 94 & 5 & 1.07 \\
4 & 19.7 & $\mathrm{NaOH}$ & 1.00 & 1 & 91 & 5 & 1.08 \\
5 & 20.0 & $\mathrm{NaOH}$ & 0.99 & 2 & 95 & 5 & 1.07 \\
6 & 19.8 & $\mathrm{NaOH}$ & 0.96 & 168 & 94 & 5 & 1.09 \\
7 & 20.0 & $\mathrm{KOH}$ & 1.00 & 1 & 84 & 5 & 1.03 \\
8 & 20.0 & $\mathrm{KOH}$ & 1.00 & 2 & 91 & 5 & 1.04 \\
9 & 20.0 & $\mathrm{KOH}$ & 0.99 & 168 & 92 & 5 & 1.05 \\
\hline TESi-PS & & & & & & & \\
10 & 19.9 & $\mathrm{LiOH}$ & 1.01 & 1 & 92 & 4 & 1.06 \\
11 & 20.0 & $\mathrm{LiOH}$ & 1.00 & 168 & 0 & - & - \\
12 & 20.0 & $\mathrm{NaOH}$ & 1.09 & 4 & 62 & 5 & 1.08 \\
13 & 20.0 & $\mathrm{NaOH}$ & 1.01 & 168 & 0 & - & - \\
\hline
\end{tabular}

${ }^{\text {a }}$ Temperature, $60^{\circ} \mathrm{C}$; heterogeneous system (TESiP-PS in THF, catalyst in $\mathrm{H}_{2} \mathrm{O}$ ). ${ }^{b}$ Determined by the GPC method. ${ }^{c}$ Degree of polymerization that was determined from the peak-top count of the GPC curve with calibration using polystyrene standards.

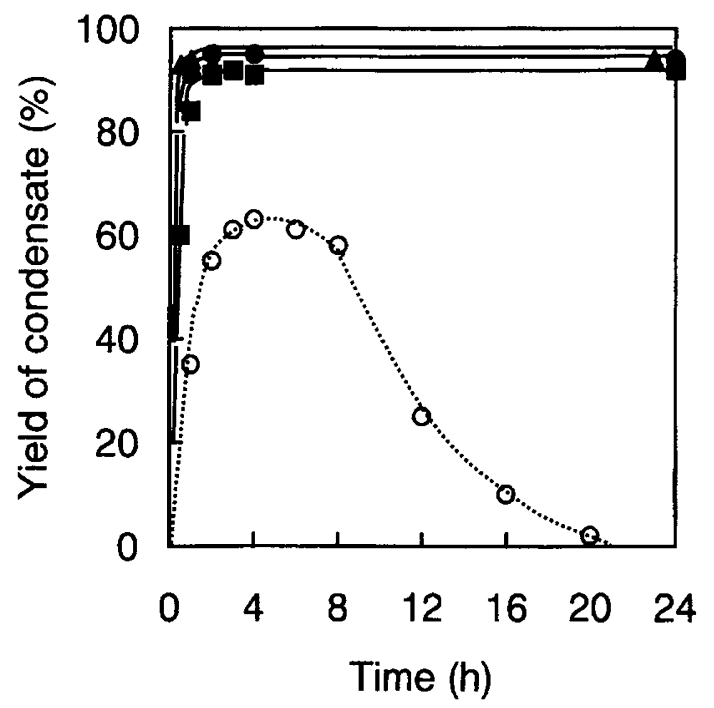

Figure 3. Time courses of condensate yields for the condensations of TESiP-PS catalyzed by $\mathrm{LiOH}(\boldsymbol{\bullet}), \mathrm{NaOH}(\bullet)$, and $\mathrm{KOH}(\boldsymbol{\bullet})$ and for the condensation of TESi-PS catalyzed by $\mathrm{NaOH}(\circ)$. Reaction conditions: solvent, THF; temperature, $60^{\circ} \mathrm{C}$; [prepolymer] $\approx$ $20 \mathrm{mmol} \mathrm{kg}^{-1}$; [cat.] $\approx 1 \mathrm{~mol} \mathrm{~kg}^{-1}$.

97\%. The results are summarized in Table III. TESiPPS was condensed almost quantitatively by $\mathrm{LiOH}$, $\mathrm{NaOH}$, or $\mathrm{KOH}$ catalyst within $1 \mathrm{~h}$. It was found that the degree of polymerization (DP) of the condensates was 5 (based on PSt standards calibration (Figure 1)) and that the molecular weight distribution was very narrow $\left(M_{\mathrm{w}} / M_{\mathrm{n}}=1.03-1.09\right)$. We confirmed that these DP values did not vary with reaction time or temperature. Conversely, in the case of TESi-PS which does not possess the spacer (trimethylene group), no condensate was detected after a long reaction time, although some condensate was obtained after a relatively short reac-

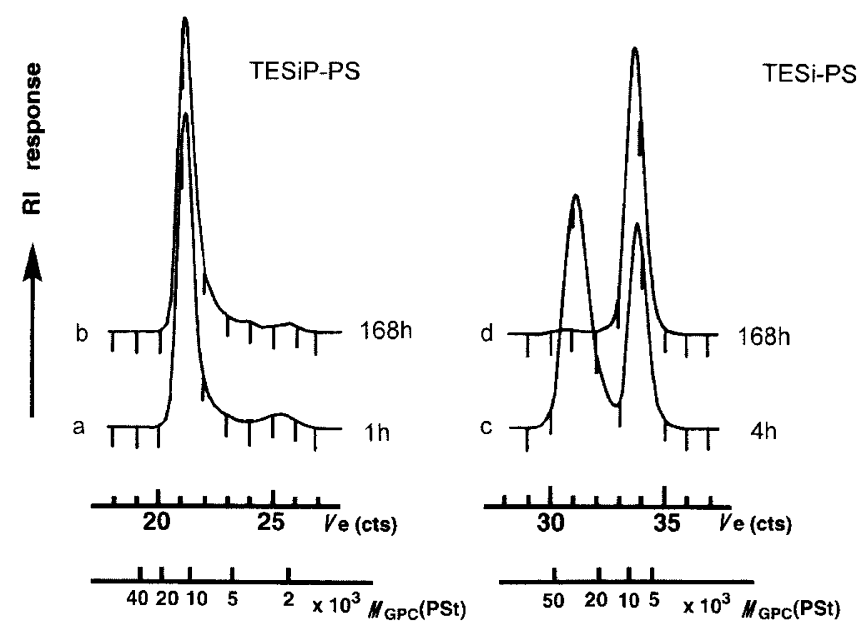

Figure 4. GPC curves of condensation products of TESiP-PS and TESi-PS. Curves a, b, c, and d are the condensation products of TESiP-PS (runs 4 and 6 in Table III) and of TESi-PS (runs 12 and 13 in Table III), respectively.

tion time. The time-dependence of condensate yield can be seen more clearly in Figure 3. In the condensations of TESiP-PS by alkali hydroxides, the curves of condensate yield-time were found to saturate at values of $c a .90 \%$ yield within $1 \mathrm{~h}$, and these values did not vary with reaction time. However, in the case of TESiPS, it was found that the condensate yield-time curve reaches a maximum point at a reaction time of $5 \mathrm{~h}$, and then the yield decreases with time. After $20 \mathrm{~h}$ no condensate was obtained.

The condensation products for TESiP-PS and TESiPS were traced with GPC. The results are shown in Figure 4. We found that the GPC profiles of products found in condensations of TESiP-PS after either a short or long reaction time are almost the same, implying that 


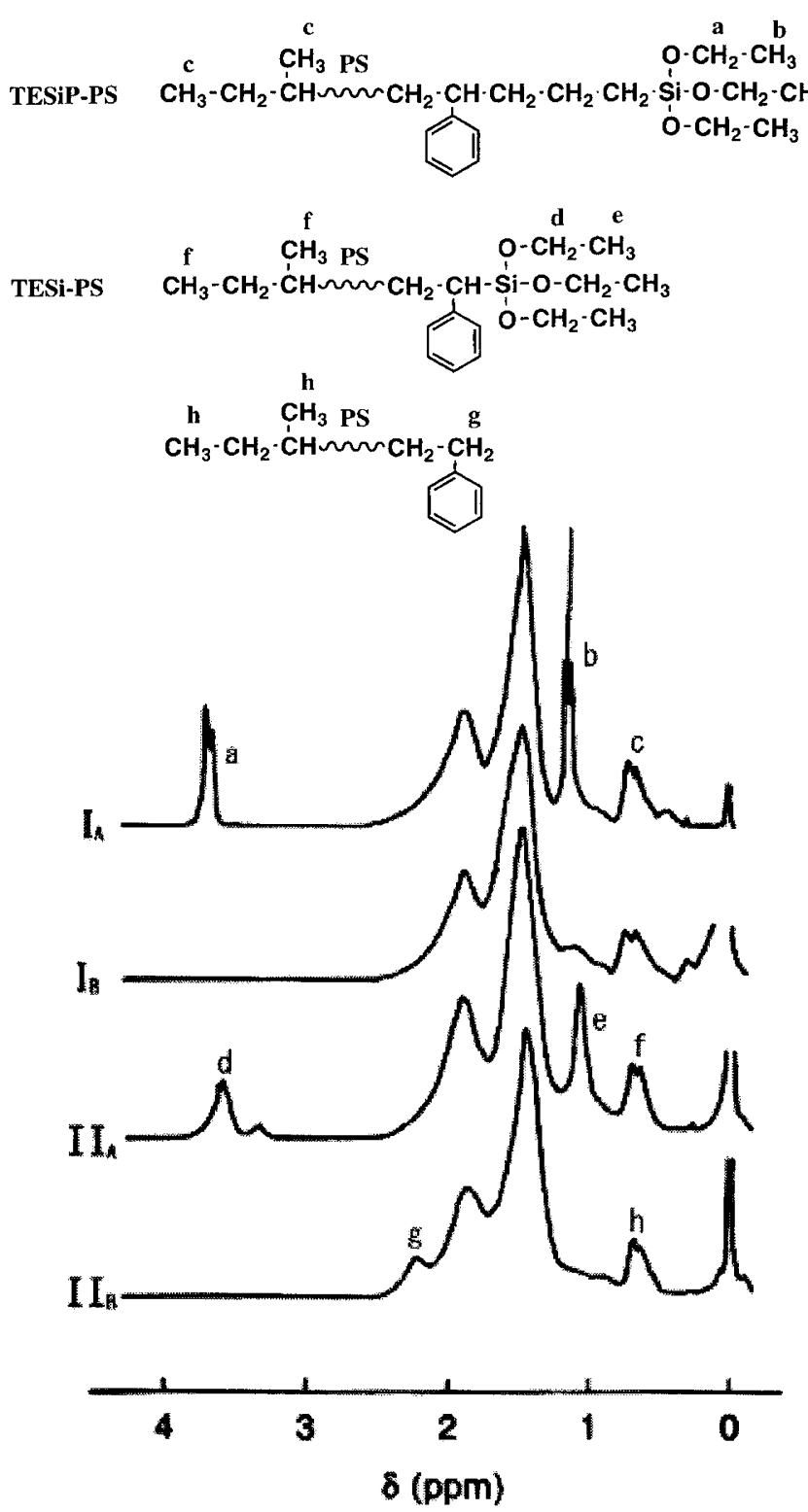

Figure 5. ${ }^{1} \mathrm{H}$ NMR spectra of the condensation products of TESiP-PS and TESi-PS: spectra $\mathrm{I}_{\mathrm{B}}$ and $\mathrm{II}_{\mathrm{B}}$ are of those obtained for the products from runs 6 and 13 in Table III, respectively; comparative spectra $\mathrm{I}_{\mathrm{A}}$ and $\mathrm{II}_{\mathrm{A}}$ are for original TESiP-PS and TESi-PS, respectively.

there is no decomposition of oligomers of TESiP-PS, once formed, even after a long reaction time. In contrast, it was confirmed by GPC profiles that oligomers of TESi-PS, once formed, decomposes completely after a long reaction time to a polymer whose molecular weight is virtually the same as that of TESi-PS.

The cause of this curious result was examined using ${ }^{1} \mathrm{H}$ NMR. ${ }^{1} \mathrm{H}$ NMR spectra of the products, which were obtained after condensation of TESiP-PS and TESiPS, catalyzed by $\mathrm{NaOH}$ for $168 \mathrm{~h}, \mathrm{I}_{\mathrm{B}}$ and $\mathrm{II}_{\mathrm{B}}$, respectively, together with comparative spectra of the original TESiP-PS $\left(\mathrm{I}_{\mathrm{A}}\right)$ and TESi-PS $\left(\mathrm{II}_{\mathrm{A}}\right)$, are shown in Figure 5.

Comparison of spectra $\mathrm{I}_{\mathrm{B}}$ and $\mathrm{I}_{\mathrm{A}}$ reveals that their resonance peaks are almost identical except for the resonance peaks of the ethoxy methyl protons ( $b$ and $\mathrm{e}, 1.14 \mathrm{ppm}$ ) and ethoxy methylene protons (a and d, $3.71 \mathrm{ppm}$ ). However, comparison of spectra $\mathrm{II}_{\mathrm{B}}$ and $\mathrm{II}_{\mathrm{A}}$ shows that spectrum $\mathrm{II}_{\mathrm{B}}$ furnishes a new signal, (g), at $2.25 \mathrm{ppm}$ due to the benzyl protons and that the peaks due to the ethoxy protons (methyl protons, (e), $1.05 \mathrm{ppm}$; methylene protons, (d), $3.71 \mathrm{ppm}$ ) disappear. The intensity ratio of the new signal (g) to that for the methyl protons (h) of the initiator fragment was 2.1 (theor, 2) : 6. These findings indicate the quantitative scission of the Si-CHPh single bond of TESi-PS.

Accordingly, the condensation reactions of TESiPPS and TESi-PS are represented in Scheme 2. We may postulate the mechanism of the cleavage reaction from the difference in behavior of the condensation reactions of TESiP-PS and TESi-PS. In nucleophilic substitution, the $\mathrm{Si}-\mathrm{CHPh}$ benzyl group of TESi-PS is assumed to be a much more superior leaving group than is the trimethylene group of TESiP-PS. That is, first, a bimolecular substitution between PS moiety and $\mathrm{OH}^{-}$, which arises from the nucleophilic attack of $\mathrm{OH}^{-}$at the ${ }^{1} \mathrm{Si}$ atom, occurs (process (a)), or second, the base draws out the $\mathrm{H}^{+}$ion from the silanol to form the silanolate and, as a consequence, the cleavage of the ${ }^{1} \mathrm{Si}-\mathrm{CHPh}$ bond of the silanolate, through formation of a silanone and benzyl anion-terminated polystyrene as intermediate, may occur (process (b)). The first assumption may be similar to the so-called $\mathrm{S}_{\mathrm{N}} 1$ or $\mathrm{S}_{\mathrm{N}} 2$ concerted process. Furthermore, the high stability of the benzyl anion probably reduces the activation energy of this elimination reaction. The second assumption is supported by the reports ${ }^{11-13}$ showing that nucleophilic substitution of a Si-leaving group proceeds via silanone formation. Eaborn et al. ${ }^{11,12}$ and Stanczyk et al. ${ }^{13}$ found that cleavage of $\mathrm{R}-\mathrm{Si}$ bonds occurs rapidly in solutions of $\mathrm{RSiMe}_{2} \mathrm{OMe}\left(\mathrm{R}=m-\mathrm{ClC}_{6} \mathrm{H}_{4} \mathrm{CH}_{2}\right.$ or $\left.\mathrm{PhC} \equiv \mathrm{C}\right)$ in $5 \mathrm{vol} \% \mathrm{H}_{2} \mathrm{O}-\mathrm{MeOH}$ containing relatively small concentrations of $\mathrm{NaOMe}$. They suggested that unimolecular dissociation of ${ }^{-} \mathrm{O}-(\mathrm{X}) \mathrm{Si}<$ species to give $\mathrm{X}^{-}$and $\mathrm{O}=\mathrm{Si}<$ (which immediately reacts with the solvent) accounts for features of the base-catalyzed cleavage of RSi bonds.

In the case of TESi-PS, the increase in hyperconjugative electron release into the benzene ring from the $\mathrm{CH}-$ ${ }^{1} \mathrm{Si}$ bond probably occurs, ${ }^{14}$ promoting the cleavage reaction of the ${ }^{1} \mathrm{Si}-\mathrm{CHPh}$ single bond. However, for TESiP-PS, the $\left(\mathrm{CH}_{2}\right)_{3}$ group may reduce such hyperconjugative effect. The reduction of this effect probably inhibits the cleavage of the $\mathrm{Si}-\mathrm{CH}_{2}$ single bond of the $\mathrm{Si}\left(\mathrm{CH}_{2}\right)_{3} \mathrm{CHPh}$ group. Thus, in the TESiP-PS related silane-coupling agents containing the $\mathrm{Si}\left(\mathrm{CH}_{2}\right)_{n} \mathrm{CHPh}$ group $(n>3)$, such a cleavage reaction probably does 
TESiP-PS

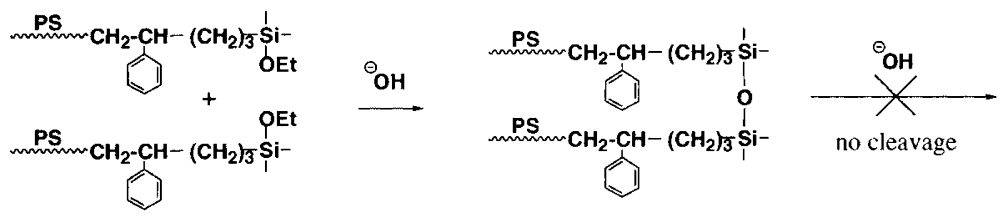

TESi-PS
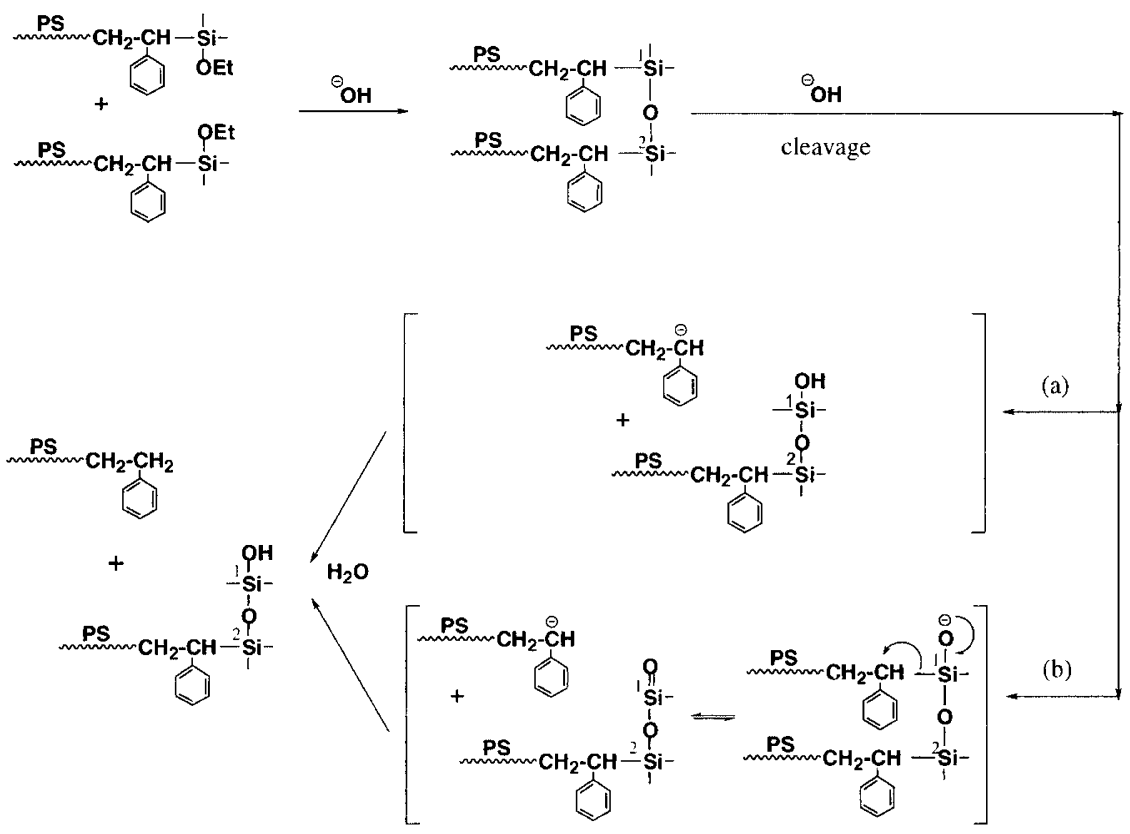

Scheme 2. Proposed reaction scheme of TESi-PS and TESiP-PS catalyzed by alkali hydroxides.

not occur, even under the strongly basic condition.

From the time-independence of the DP values for the TESiP-PS condensates, we may propose the presence of possible cyclic molecular structures for the condensates (to be reported separately). Further work (for example, a ${ }^{29} \mathrm{Si}$ CP MAS NMR study ${ }^{15}$ ) is highly desirable, in order to determine which cyclic structure is predominantly stabilized in solution and in the solid state. Recently, Izawa et al. ${ }^{16}$ successfully used SAXS spectra to detect the aggregates of alkylalkoxysilane in ethanol. They also used 2D correlation $\mathrm{GPC}^{17}$ to confirm the correlation between the silanecoupling monomer-polymer aggregates, thus confirming the presence of the aggregates.

Thus, in the case of the TESiP-PS condensates, the presence of aggregates brought about by the hydrophobic polystyrene portions is possible. Moreover, steric hindrance by the bulky polystyrene moiety isolates the $\mathrm{SiOH}$ groups, thus preventing further reaction.

\section{CONCLUSIONS}

A polymeric silane coupling agent, TESiP-PS, which can be condensed by each of base and acid catalysts, has been developed. Synthesized TESiP-PS possesses a high functionality and a narrow molecular weight distribution, and its molecular weight is easily controlled, since the polymeric moiety is prepared by living anionic polymerization.

The condensation of TESiP-PS proceeds quantitatively in the presence of either base or acid catalysts and affords oligomers (TESiP-PS) ${ }_{a}$ with a degree of polymerization of 5.0 and a narrow molecular weight distribution. In the condensation, the oligomers of TESiP-PS do not undergo cleavage in the presence of base catalyst, although the oligomers of TESi-PS are cleaved completely. This difference verifies that the trimethylene spacer group is effective for perfect suppression of the cleavage reaction.

\section{REFERENCES}

1. E. P. Plueddemann, "Silane Coupling Agents", 2nd ed, Plenum Publishing, New York, N.Y., 1990.

2. T. E. Long, L. W. Kelts, S. R. Turner, J. A. Wesson, and T. H. Mourey, Macromolecules, 24, 1431 (1991).

3. M. Takaki, K. Suzuki, and T. Mano, Kobunshi Ronbunshu, 48, 
171 (1991).

4. M. Takaki, K. Suzuki, Y. Kondo, and J. Oku, Polym. J., 23, 917 (1991).

5. K. Suzuki, G. Katsumura, Y. Kondo, J. Oku, and M. Takaki, Kobunshi Ronbunshu, 49, 825 (1992).

6. K. Suzuki, M. Esaki, A. Misawa, and M. Takaki, Kobunshi Ronbunshu, 51, 11 (1994).

7. T. Altares, D. P. Wyman, V. R. Allen, and K. Meyersen, J. Polym. Sci., Part A: Polym. Chem., 3, 4131 (1965).

8. J. C. Meunier and R. Van. Leemput, Makromol. Chem., 142, 1 (1971).

9. M. Takaki, R. Asami, and M. Ichikawa, Macromolecules, 10, 850 (1977).

10. M. Takaki, R. Asami, and Y. Kuwata, Polym. J., 11, 425 (1979).
11. Z. H. Ajube, J. Chojnowski, C. Eaborn, and W. Stanczyk, J. Chem. Soc., Chem. Commun., 493 (1983).

12. C. Eaborn and W. Stanczyk, J. Chem. Soc., Perkin Trans., 2, 2099 (1984).

13. W. Stanczyk and M. Scibiorek, Makromol. Chem., Rapid Commun., 4, 703 (1983).

14. C. Eaborn and S. H. Parker, J. Chem. Soc., 939 (1954).

15. G. E. Maciel and D. W. Sindorf, J. Am. Chem. Soc., 102, 7606 (1980).

16. K. Izawa, T. Ogasawara, H. Masuda, H. Okabayashi, M. Monkenbush, and C. J. O'Connor, Colloid Polym. Sci., 280, 725 (2002)

17. K. Izawa, T. Ogasawara, H. Masuda, H. Okabayashi, and I. Noda, Macromolecules, 35, 92 (2002). 